\title{
Unique strategy using a standard double lumen tube during one lung ventilation for successful intraoperative airway management in patients with a giant bulla on the ventilated side: case series
}

\author{
Izumi Kawagoe ${ }^{1}$ (D) Masakazu Hayashida ${ }^{1} \cdot$ Daizoh Satoh $^{1} \cdot$ Osamu Kudoh $^{1} \cdot$ Masataka Fukuda $^{1}$. \\ Tsukasa Kochiyama ${ }^{1}$. Jun Kishii ${ }^{1}$. Chieko Mitaka ${ }^{1}$
}

Received: 3 March 2021 / Accepted: 20 August 2021 / Published online: 26 August 2021

(c) Japanese Society of Anesthesiologists 2021

\begin{abstract}
Giant lung bullae are usually seen in patients with severe chronic obstructive pulmonary disease. Over time, air trapping leads to severe dyspnea and $\mathrm{CO}_{2}$ accumulation. In severe cases, overinflation and rupture of the bulla can cause secondary life-threatening tension pneumothorax. Since positive pressure ventilation exerts deleterious effects on the bulla, general anesthesia is always challenging in patients with giant bullae. We encountered remarkable intraoperative hypercapnia and decreased tidal volume in a 58-year-old male patient with bilateral bullae who underwent right upper bullectomy, due to overinflation of a bulla located in the upper lobe of the ventilated side. Through this experience, to avoid further overinflation, we devised an original, unique and simple airway management strategy using a standard double lumen tube (DLT), which only requires slightly deeper advancement of the DLT to achieve selective lobar blockade during one lung ventilation (OLV). Following the first case, we used this strategy in a 48-year-old male patient who underwent left giant bullectomy, resulting in successful airway management without overinflation during OLV. We recommend our strategy as an option for successful intraoperative airway management during OLV in select bullectomy patients with bilateral giant bullae.
\end{abstract}

Keywords Giant bulla resection - Airway management $\cdot$ Double lumen tube $\cdot$ Selective lobar blockade

\section{Introduction}

Izumi Kawagoe

ikawago@juntendo.ac.jp

Masakazu Hayashida

mhaya@juntendo.ac.jp

Daizoh Satoh

da-sato@juntendo.ac.jp

Osamu Kudoh

kudora@juntendo.ac.jp

Masataka Fukuda

msfukuda@juntendo.ac.jp

Tsukasa Kochiyama

tkouchi@juntendo.ac.jp

Jun Kishii

j-kishii@juntendo.ac.jp

Chieko Mitaka

c-mitaka@juntendo.ac.jp

1 Department of Anesthesiology and Pain Medicine, Juntendo University School of Medicine, 2-1-1 Hongo, Bunkyo-ku,

Tokyo 113-8421, Japan

\section{Background}

A bulla is an abnormal collection of air within the lung parenchyma, which is defined pathologically as a dilated air space more than $2 \mathrm{~cm}$ in diameter [1], and as a giant bulla when it occupies more than one third of the lung volume [2]. Giant lung bullae are located unilaterally or bilaterally, typically in the upper lung lobe, usually in patients with severe chronic obstructive pulmonary disease (COPD). Over time, air trapping leads to severe dyspnea and $\mathrm{CO}_{2}$ retention and, sometimes, life-threatening secondary tension pneumothorax. Since positive pressure ventilation (PPV) can cause further distension of bullae, general anesthesia is challenging in these patients.

Here, we describe our experience with a case where we devised a unique strategy of one lung ventilation (OLV) in a patient with bilateral lung bullae, and another similar case in whom we preemptively used the strategy. The institutional review board of our hospital approved these case 
presentations, and the patients gave written informed consent for publication of this case report and any accompanying images.

\section{Case presentations}

\section{Case 1}

A 58-year-old male patient, $174 \mathrm{~cm}$ tall, weighing $69 \mathrm{~kg}$, was scheduled for right upper lobe bullectomy due to recurrent infection in the bulla for 6 months. He had a 38 pack-peryear smoking history with COPD (Fig. 1a, b). The results of preoperative examinations are shown in Table 1.
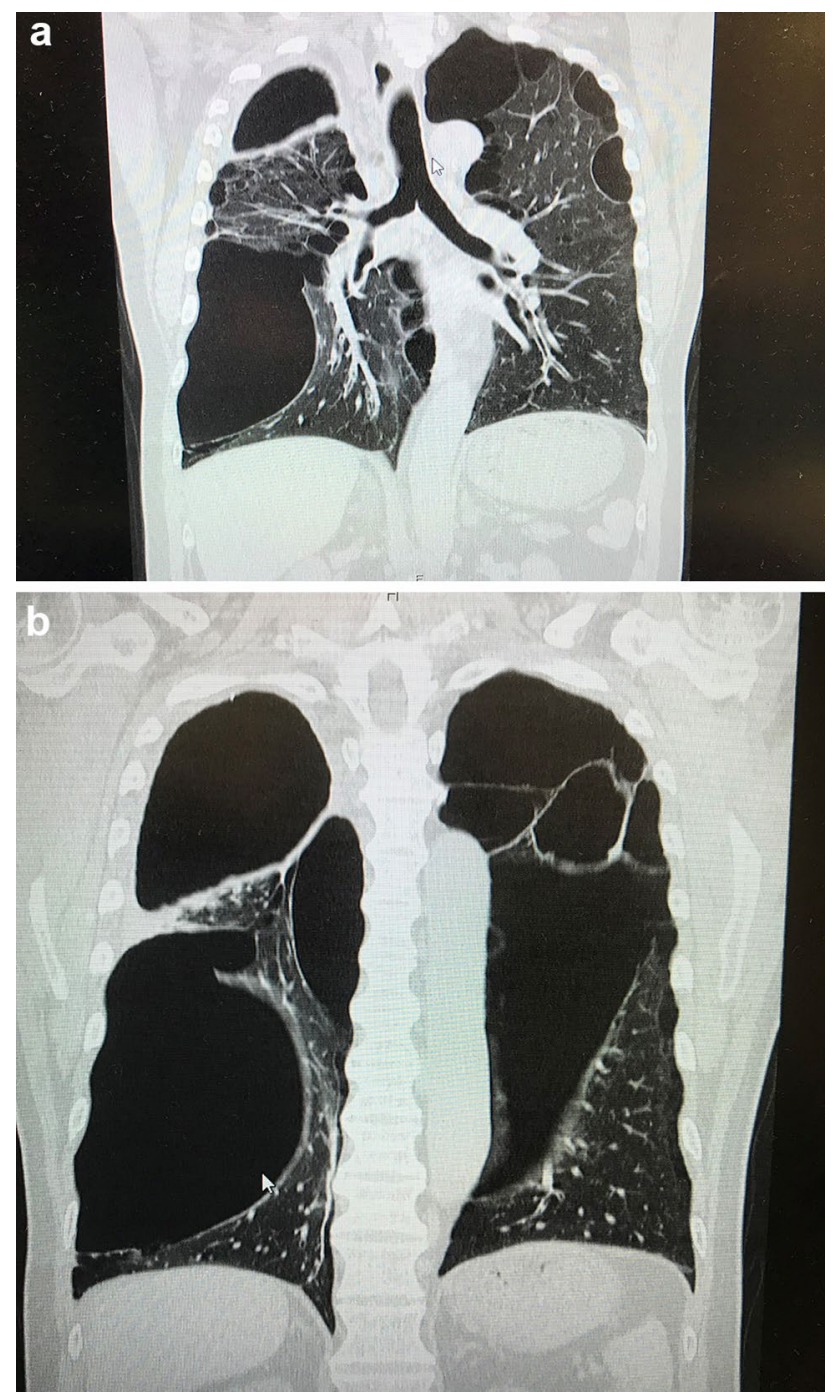

Fig. 1 a, b bilateral bulla in Case 1. Bilaterally, the upper lobes were occupied with giant bullae. The left side was ventilated in Case 1
Preoperatively, after epidural catheter insertion, general anesthesia was induced and maintained with propofol (2-3 $\mathrm{mcg} / \mathrm{mL}$ by target-controlled infusion) and remifentanil $(0.1-0.25 \mathrm{mcg} / \mathrm{kg} / \mathrm{min})$, and muscle relaxation was achieved with a $50 \mathrm{mg}$ bolus of rocuronium. After adequate muscle relaxation, a 37F left-sided double lumen tube (DLT) (Blueline/Portex, Smiths Medical, Minneapolis, USA) was inserted into his trachea under laryngoscopy and was advanced into his left main bronchus under bronchoscopic guidance. The left-sided DLT was accurately positioned so that the tip of the bronchial lumen remained just above the secondary carina of the left bronchus, so that both the left upper and lower lobes could be fully ventilated. The initial tidal volume during left-sided OLV was more than $350 \mathrm{~mL}$ with pressure controlled ventilation (PCV) delivered at the settings shown in Table 1.

Approximately halfway through the surgery, end-tidal $\mathrm{CO}_{2}\left(\mathrm{EtCO}_{2}\right)$ increased to $90 \mathrm{mmHg}$, and tidal volume decreased to below $100 \mathrm{~mL}$. After ruling out DLT malposition, pneumothorax and airway obstruction, we assumed that some of the inspiratory volume passed into the bulla in the left upper lobe, with a simultaneous decrease in effective ventilation. Further application of PPV could have led to potential rupture of the bulla. Hence, as a rescue procedure, we decided to advance the bronchial lumen of the left-sided DLT more deeply, and inserted it into the left lower bronchus under bronchoscopic guidance, placing it above the next bifurcation without causing damage, to achieve selective left lower lobe ventilation (Fig. 2a). Subsequently, the effective tidal volume recovered to $300 \mathrm{~mL}$, and $\mathrm{EtCO}_{2}$ decreased to $55 \mathrm{mmHg}$ at 60 min after the rescue procedure (Fig. 3). The patient was extubated immediately postoperatively and shifted to the intensive care unit (ICU). After staying in the ICU overnight, he was discharged from the hospital in an ambulatory condition on the 13th postoperative day with home oxygen therapy.

\section{Case 2}

A 48-year-old male patient, $182 \mathrm{~cm}$ tall, weighing $79 \mathrm{~kg}$, was scheduled to undergo resection of a giant bulla in the left upper lobe. His giant bulla repeatedly developed infections, resulting in abscess formation (Fig. 4a, b). He had a family history of bullous disease and an episode of spontaneous pneumothorax at the age of 23 years. He had a 28 pack-per-year smoking history, and well-controlled asthma with $5 \mathrm{mg}$ steroid and $40 \mathrm{mg}$ theophylline. The results of preoperative examinations are shown in Table 1. Anesthesia induction and maintenance were performed in 
Table 1 Preoperative examination and perioperative data

\begin{tabular}{|c|c|c|c|}
\hline & & Case 1 & Case 2 \\
\hline Preoperative pulmonary function & $\begin{array}{l}\text { Vital capacity }(\mathrm{L}) \\
\text { (\% of expected value) } \\
\text { Forced expiratory vol- } \\
\text { ume (FEV)1.0 (L) } \\
\text { (\% of value expected) } \\
\text { FEV } 1.0 \%\end{array}$ & $\begin{array}{l}1.9 \\
(45) \\
1.07 \\
(31) \\
56.3\end{array}$ & $\begin{array}{l}3.17 \\
(65.4) \\
1.03 \\
(25.4) \\
32.5\end{array}$ \\
\hline $\begin{array}{l}\text { Preoperative arterial blood gas evaluation } \\
\text { (under room air) }\end{array}$ & $\begin{array}{l}\mathrm{pH} \\
\mathrm{PaCO}_{2}(\mathrm{mmHg}) \\
\mathrm{PaO}_{2}(\mathrm{mmHg}) \\
\mathrm{BE}(\mathrm{mEq} / \mathrm{L}) \\
\mathrm{HCO}_{3}(\mathrm{mEq} / \mathrm{L}) \\
\mathrm{SpO}_{2} \%\end{array}$ & $\begin{array}{l}7.386 \\
48 \\
72 \\
2.3 \\
28.2 \\
94.7\end{array}$ & $\begin{array}{l}7.5 \\
33 \\
93 \\
2.8 \\
25.3 \\
97.8\end{array}$ \\
\hline $\begin{array}{l}\text { Intraoperative ventilator settings } \\
\text { for pressure-controlled ventilation } \\
\text { during OLV }\end{array}$ & $\begin{array}{l}\text { Peak pressure }\left(\mathrm{cmH}_{2} \mathrm{O}\right) \\
\text { Respiratory rate }(/ \mathrm{min}) \\
\text { Inspiration to expiration } \\
\text { ratio } \\
\text { Positive end-expiratory } \\
\text { pressure }\left(\mathrm{cmH}_{2} \mathrm{O}\right)\end{array}$ & $\begin{array}{l}23-25 \\
12-14 \\
1: 1.5-2.5 \\
4\end{array}$ & $\begin{array}{l}23 \\
14 / \min \\
1: 2 \\
4\end{array}$ \\
\hline Anesthesia /surgical duration (min) & & $264 / 221$ & $413 / 374$ \\
\hline
\end{tabular}

FEV 1.0 forced expiratory volume/ vital capacity almost the same way as described for Case 1. A 37F rightsided DLT (Shiley/Mallinckrodt, Medtronic Inc., Minneapolis, USA) was inserted into his trachea above the carina under laryngoscopic visualization, and further inserted into the right main bronchus under bronchoscopic guidance. Based on our previous experience in Case 1, unusually deep placement of the right-sided DLT to just above the right middle lobe and basal bronchi was performed, ensuring that the lateral hole was not aligned with the upper bronchus, so that the giant bulla located in the right upper lobe would not be ventilated (Fig. 2b). During OLV via the right middle and lower lobes, the initial tidal volume was approximately $350 \mathrm{~mL}$ with PCV at the settings shown in Table 1. Ventilator settings and tidal volume remained unchanged throughout the entire OLV period. Maximal $\mathrm{PaCO}_{2}$ remained at $55 \mathrm{mmHg}$ during OLV in Case 2. The patient had a good postoperative course and was discharged on the 14th postoperative day.

\section{Discussion and conclusions}

Since the diameter of the DLT is smaller than that of a standard single lumen tube, plateau pressure and peak inspiratory pressure increase by $24 \%$ and $55 \%$, respectively, with OLV compared to bilateral ventilation [3]. Even during non-thoracic surgery under general anesthesia with PPV, air trapping and further expansion of bullae can occur. Hence, anesthesiologists sometimes avoid PPV in patients with giant bullae. Partial blockade of the lung lobe occupied by the bulla
[4] and spontaneous breathing have also been described as alternative management strategies [5]. Previous reports described the use of a combination of a left-sided DLT and bronchial blocker (BB) to perform selective blockade of the right upper lobe in a patient with a giant right-sided bulla who underwent non-thoracic surgery, and in thoracic abscess patients who underwent thoracic surgery [5, 6]. Another report described the performance of volume reduction surgery (VRS) using video-assisted thoracic surgery (VATS) in a non-intubated, spontaneously breathing subject under light sedation [4].

In our case series, successful airway management was achieved by inserting the DLT slightly deeper than the conventional placement used to occlude the upper lobe bronchus. OLV with ventilation of the lower lobes is typically associated with hypoxemia in patients without a giant bulla. Hence, hypothetically, hypoxia would be similarly expected during OLV in patients with bullae. Conversely, however, OLV with our strategy to limit air leakage into the bulla might lead to maintenance or improvement of oxygenation due to reduction in ventilation/perfusion mismatch. Our novel strategy also reduces the risk of bulla rupture due to overinflation during PPV.

In our first case, we had not anticipated severe intraoperative hypercapnia and decreased tidal volume because we underestimated the degree of preoperative respiratory dysfunction based on the patient's good daily activity levels. We hypothesized that the changes occurred due to reduction of effective ventilation secondary to expansion of the bulla in the left upper lobe, which indicated the urgent need 

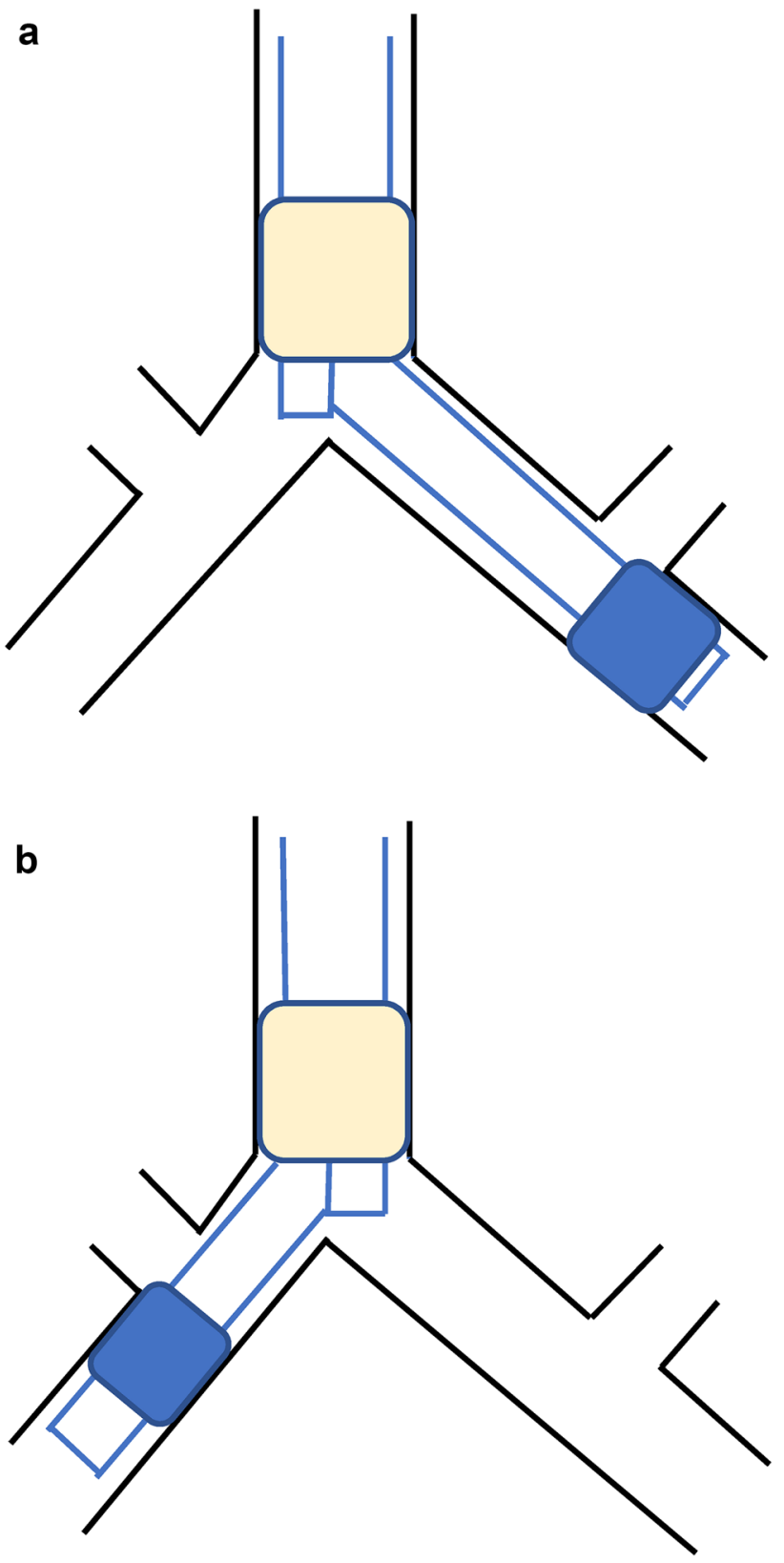

Fig. 2 a schematic showing advancement of the bronchial cuff of the left-sided DLT up to the left lower bronchus in Case 1. A Blue line double lumen tube (DLT) (Smiths Medical) was used in Case 1. b schematic showing of advancement of the bronchial cuff of the rightsided DLT up to the right bronchus intermedius in Case 2. A broncho-cath DLT (Medtronic) was used in Case 2 for avoiding further overinflation of the bulla. Initially, we explained the emergency situation to the surgeons and asked them to allow bilateral ventilation to reduce inspiratory pressure. Although we considered performing selective blockade of the upper lobe with a BB combined with the DLT, this was considered difficult with the type of DLT being used in the patient due to incompatibility with the connector. Fortunately, however, we realized that it was possible to insert the DLT more deeply into the left lower bronchus, achieving selective upper lobe blockade without using a bronchial blocker, which was a strategy that we had considered in advance as a worst case scenario.

Following our experience in the first case, we planned to perform selective blockade of the upper lobe in the second case. We preoperatively confirmed the feasibility of using the right-sided DLT in this case by measuring the length of the bronchial balloon directly and that of the right intermedius bronchus from a preoperative CT scan. The DLT used in Case 2 was also confirmed to be usable with a BB. The measurements indicated that the length of the bronchial balloon of the DLT was sufficient for it to be accommodated in his intermedius bronchus, without occlusion of the middle and lower lobe bronchus. Although we did consider the alternative method of inserting a left-sided DLT into the right main bronchus, we considered this too difficult due to the anticipated mismatch between the form of the left-sided DLT and angulation of the right main bronchus. With our strategy, the patient's tidal volume and oxygenation during OLV remained stable intraoperatively despite the more than $6 \mathrm{~h}$ surgical duration. It should be cautioned that the safety and reliability of our strategy depends on the individual patient's height and bronchial anatomy, particularly the length of left main bronchus in right lung surgery, the kind of DLT product, and the anesthesiologist's skill, since excessively deep insertion of the DLT can cause bronchial damage. Hence, although we cannot strongly recommend our technique in all cases, it might be a valuable alternative in certain cases. Particularly in left-sided pulmonary surgery cases, our strategy of using a right-sided DLT might be acceptable and recommendable from a safety and utility perspective.

In conclusion, this report highlights the utility of our unique strategy, which only requires slightly deeper than usual advancement of the DLT, for successful airway management during anesthesia in select patients with giant bullae in the upper lobe of the ventilated lung. 
Fig. 3 Trend of $\mathrm{EtCO}_{2}$ in Case 1. $\mathrm{EtCO}_{2}$ increased to $90 \mathrm{mmHg}$ at $120 \mathrm{~min}$ after starting OLV. Following performance of our strategy at $120 \mathrm{~min}, \mathrm{EtCO}_{2}$ decreased to below $55 \mathrm{mmHg}$. OLV was discontinued at approximately $180 \mathrm{~min}$

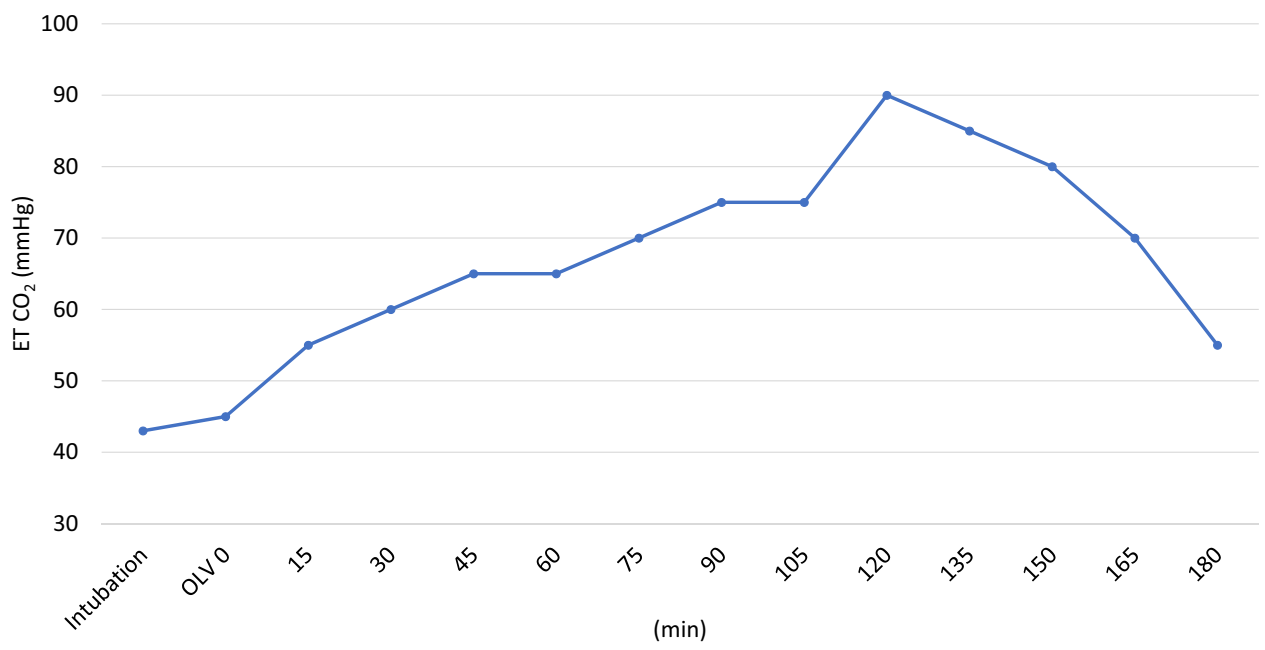

(min)
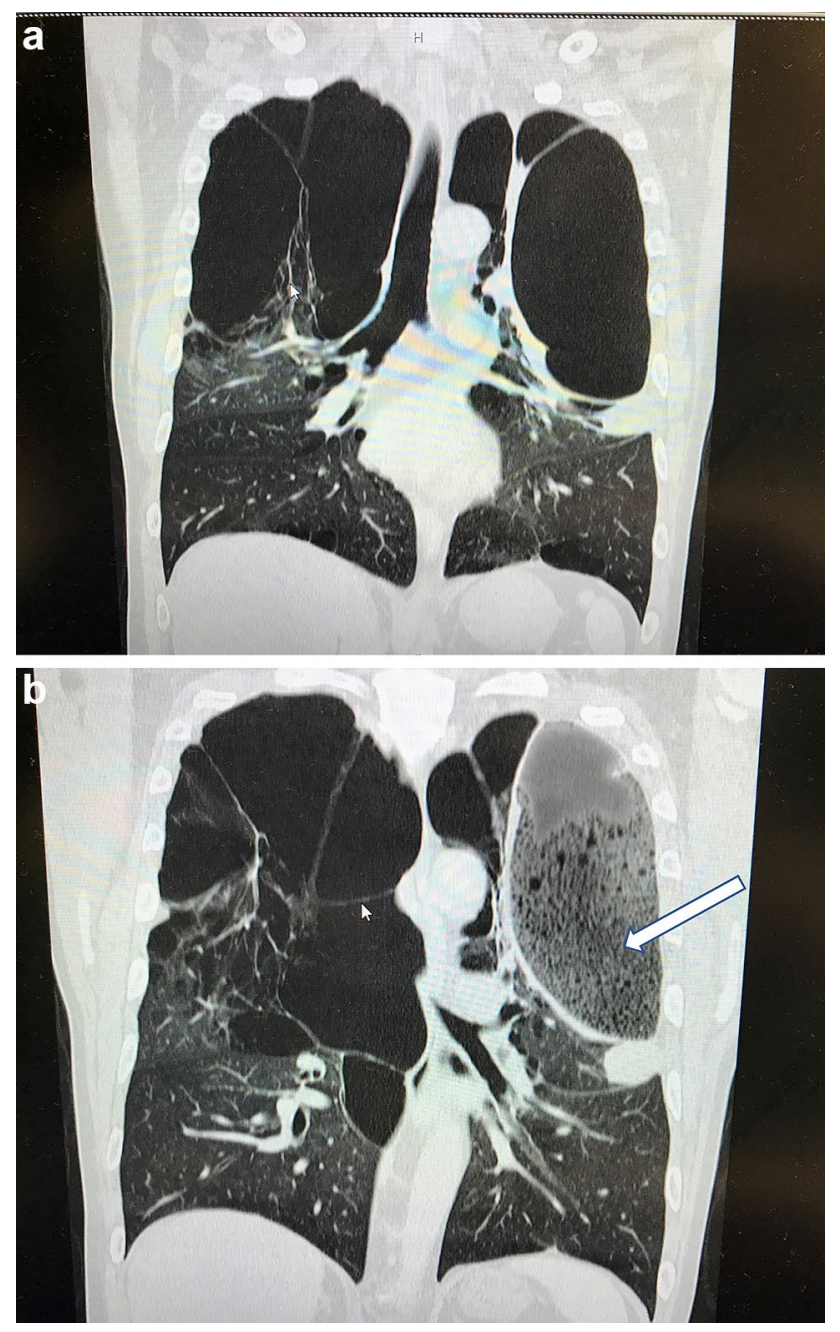

Fig. 4 a, b Bilateral bullae in Case 2. The right upper lobe was occupied with a giant bulla, which was the ventilated side in Case 2. The left upper lobe was occupied with a giant bulla that included the abscess to be resected. Arrow: abscess in the left upper lobe
Acknowledgements None.

Author contributions Izumi Kawagoe, Tsukasa Kochiyama, Osamu Kudo, Daizoh Satoh, Masataka Fukuda, Jun Kishii, Masakazu Hayashida and Chieko Mitaka performed anesthesia and postoperative care. Izumi Kawagoe wrote the manuscript. All authors read and approved the final manuscript.

\section{Funding None.}

Data availability The datasets related to this report are available from the corresponding author on reasonable request.

\section{Declarations}

Conflict of interest All the authors declare that they have no competing interests.

Ethical approval The institutional review board of our hospital approved this case presentation.

Consent for publication The patient gave written informed consent for publication of this case report and any accompanying images.

\section{References}

1. Stern EJ, Frank MS. CT of the lung in patients with pulmonary emphysema: diagnosis, quantification, and correlation with pathologic and physiologic findings. AJR Am J Roentgenol. 1994;162:791-8.

2. Shah NN, Bhargava R, Ahmed Z, Pandey DK, Shameem M, Bachh AA, Dar KA. The vanishing lung. CJEM. 2007;9(170):233-4.

3. Saegedi LL, Bardoczky GI, Engelman EE, d'Hollander AA. Airway pressure changes during one-lung ventilation. Anesth Analg. 1997;84:1034-7.

4. Theodare B, Nisssy VV, Sahajanandan R, Mariappan R. Anesthetic challenges of a patient with the communicating bulla coming for nonthoracic surgery. Ann Card Anaesth. 2018;22:200-2. 
5. Lan L, Li J, Xu X, Cen Y. Lung Volume Reduction Under Spontaneous Ventilation in a Patient with Severe Emphysema. Am J Case Rep. 2019;20:125-30.

6. Granell M, Pereira F, Peris R, Dolz L, Almenara N, de Andrés J. Lung abscess isolation using double lumen tubes combined with bronchial blockers: a case series. J Cardiothorac Vasc Anesth. 2017;31(1):S51-2.
Publisher's Note Springer Nature remains neutral with regard to jurisdictional claims in published maps and institutional affiliations. 